\title{
LXX. On the products of the decomposition of cuminate of ammonia by heat
}

\author{
Mr. Frederick Field
}

To cite this article: Mr. Frederick Field (1847) LXX. On the products of the decomposition of cuminate of ammonia by heat, Philosophical Magazine Series 3, 31:210, 459-467, DOI: $10.1080 / 14786444708645892$

To link to this article: http://dx.doi.org/10.1080/14786444708645892

册 Published online: 30 Apr 2009.

Submit your article to this journal \lceil

Џll Article views: 3

Q View related articles $₫$ 
The acid had a density of 1.424 at $60^{\circ}$; it began to boil at $250^{\circ}$, and distilled over perfectly colourless and unchanged; towards the end, when slight decomposition commenced, the temperature rose to $260^{\circ}$.

Five or six ounces of very weak acid, of the density of $1 \cdot 180$, were introduced into a retort and kept heated just below its boiling-point for two or three hours; the heat was increased from time to time so as to make it boil briskly, and a thermometer introduced through the tubulure; when that which remained in the retort boiled uniformly at $250^{\circ}$, the heat was withdrawn and it was allowed to cool.

When the specific gravity of this acid was taken, it was found to be close upon that of the tetra-hydrate, but not exactly; probably if I had operated upon a large quantity, and carried it on for a longer time, it would have been more so; as found, its density was 1.412 instead of 1.424 , which would make a difference of rather less than $1 \frac{1}{2}$ per cent. deficiency in the acid.

This is, I have no doubt, the proper hydrate of nitric acid, $\mathrm{HO}, \mathrm{NO}_{5}+3 \mathrm{HO}$, as it is generally considered; and as Dr. Dalton correctly observed, acids which are either stronger or weaker than this acid, are brought to this strength by continued ebullition, the former losing acid and the latter water.

LXX. On the Products of the Decomposition of Cuminate of Ammonia by Heat. By Mr. Frederick Field*.

THE peculiar mode of decomposition which the ammonia 1 salts of inorganic acids exhibit when exposed to the action of heat, occurs likewise in the ammonia compounds of organic acids, although the results in the latter instances are usually of a more complicated nature. In most of these cases a formation of water takes place, the hydrogen of which is derived from the volatile alkali, while the acid furnishes the oxygen, the residue of which combines in a more intimate manner with the nitrogen of the ammonia. In decompositions, however, of inorganic compounds this reduction seems to be carried at once as far as it can go, the whole of the hydrogen contained in the ammonia being converted into water; while in organic salts this hydrogen is eliminated only by degrees, an intermediate body being produced between the original ammonia salt and the final product of the decomposition. Thus we find that nitrite and nitrate of ammonia, when exposed to heat, are at once converted into water, and respectively into nitrogen and nitrous oxide. Oxalate of ammonia,

* Communicated by the Chemical Society; having been read June 7, 1847. 
on the other hand, if submitted to a gentle heat, loses only two equivalents of water, the residue of both base and acid combining to form oxamide, and only by a strong and brisk application of heat Dobereiner converted it into cyanogen, the rest of the hydrogen being eliminated in the form of water.

The dry distillation of oxalate of ammonia thus affords the prototypes of two series of compounds, which may arise from ammoniacal salts by the elimination of two or four equivalents of water respectively. There are few cases, however, in which the decomposition of ammoniacal salts have been carefully studied, and the instances in which we are acquainted with the representative of the two types are exceedingly scarce. We are indeed intimate with a very great number of amidogen compounds analogous to oxamide (fumaramide, salicylamide, succinamide, anisylamide, \&c.), but only few of these have been obtained from ammoniacal salts by the action of heat. The greatest number of these bodies were produced by the change most compound æthers suffer under the influence of ammonia, a beautiful mode of decomposition pointed out first by Professor Liebig in the transformation of oxalate of ethyl into oxamide, or by the action of gaseous ammonia on other substances related in some manner with the acid: thus was chloride of benzoyle converted into benzamide by Wöhler and Liebig, and lately lactide into lactimide by Pelouze.

As yet, however, the members of the second class, those compounds standing to other acids in the same relation as cyanogen to oxalic acid, are very rare. From a beautiful experiment of Pelouze, we know that the vapour of formiate of ammonia, when passed through a red-hot tube, is converted into water and hydrocyanic acid. In their investigation on the radical of benzoic acid, Wöhler and Liebig obtained a peculiar oil by the action of heat on benzamide, which at that time they did not study more closely. The same body was at a later period obtained in the dry distillation of benzoate of ammonia, and fully examined by Fehling, who found that this interesting substance, to which he gave the name benzonitrile, has the composition $\mathrm{C}_{14} \mathrm{H}_{5} \mathrm{~N}$, and is produced from benzoate of oxide of ammonium, exactly in the same manner as cyanogen and prussic acid are formed respectively from oxalate or formiate of ammonia. These facts did not long remain isolated. Schlieper, in an excellent examination he has lately published on the products of oxidation of gelatine by chromic acid, discovered that in these reactions, among other products, the body $\mathrm{C}_{10} \mathrm{H}_{9} \mathrm{~N}$ is formed, valeronitryle or valerianate of ammonia-4 equivs. of water.

The members of this class acquire every day a greater degree of importance. A remarkable paper, read before the 
Chemical Society a short time since by Dr. Kolbe and Mr. Frankland, has indeed opened a most interesting connexion between these bodies and another class of substances, which hitherto have been obtained by very different processes. The conversion of cyanide of ethyl into metacetonic acid by means of alkalies and acids, seems to indicate that cyanide of ethyl is nothing else than metacetonitryle. This experinent is likely to be of great importance, for it is exceedingly probable that the whole class of substances alluded to must be considered as a class of cyanogen compounds. It is evident that similar considerations may be applied to cyanide of methyl and cyanide of amyl, lately described by Balard ; and the conversion of these cyanides respectively into acetic and caproic acids, which we have a right to anticipate on treating them with alkalies or acids, will prove that these compounds are the nitriles of acetic and caproic acids-acetonitryle and capronitryle-which as yet have not been obtained by the action of heat on the ammoniacal salts of these acids.

The following experiments on the action of heat on cuminate of ammonia have been made with the hope of contributing to the history of the nitryles, or organic cyanides, as they perhaps should be more correctly designated.

The cuminic acid employed in my experiments was prepared by the action of solid hydrate of potash on oil of cumin, and the product perfectly freed from the least traces of cymol which it might possibly contain by precipitating the potash salt by hydrochloric acid, dissolving the precipitated cuminic acid in ammonia, reprecipitating by hydrochloric acid, and crystallizing from water. The acid was then dissolved in strong ammonia, and the solution subjected to heat. The first portions which passed over, although consisting chiefly of water and ammonia, together with cuminate of ammonia, which is always carried over with the steam, presented more or less an opalescent appearance, indicative of traces of the oil. On evaporating the solution in the retort to dryness, a portion of the salt is decomposed, ammonia is evolved, and cuminic acid condenses in beautiful plates upon the sides and neck of the retort, separation going on even on raising the temperature; but simultaneously another decomposition takes place, water is eliminated, in consequence of which there are produced a peculiar white crystalline body, difficultly soluble in water, and subsequently a colourless oil of a most fragrant odour; although the operation may seem very simple, experience alone teaches the proper regulation of temperature necessary to obtain these two bodies.

Cuminamide.-Observing in my first experiments evolution of ammonia and sublimation of cuminic acid on heating cu- 
minate of ammonia, I thought that by heating it under pressure, the ammonia then not being able to escape, the desired change might be effected. Accordingly a portion of the salt was placed in a strong glass tube, and after sealing the other end, gradually heated in an oil-bath to nearly the boilingpoint of the oil, and allowed to cool. On cooling the mass appeared to have been completely fused, but perfectly solid and of a highly-crystallized texture. On examination it was found to be insoluble in cold water and ammonia, but very soluble in hot water, from which it solidified into a crystalline mass as the temperature cooled; this alone sufficiently indicated that a complete change had been effected, the cuminate of ammonia being readily soluble in cold water. In order to ascertain the nature of the change it was dissolved in hot water, and weak ammonia added to dissolve any cuminic acid that might be mixed with it, and crystallized; the crystals were separated by filtration, and once more dissolved in a hot weak solution of ammonia, from which they separated on cooling in brilliant white crystalline plates, similar in appearance to benzamide. These were dried at $212^{\circ}$ in a water-bath, and analysed in the usual manner.

I. $0 \cdot 174 \mathrm{grm}$. of substance burnt with oxide of copper yielded 0.470 of carbonic acid and 0.128 of water.

II. $0.248 \mathrm{grm}$. yielded 0.670 of carbonic acid and 0.181 of water.

III. To estimate the nitrogen, $0.287 \mathrm{grm}$. ignited with sodalime yielded 0.390 of ammonio-chloride of platinum*.

From these analytic results the following per-centages are obtained :-

$\begin{array}{lrrr} & \text { I. } & \text { II. } & \text { III. } \\ \text { Carbon . . } & 73 \cdot 66 & 73 \cdot 67 & \\ \text { Hydrogen : } & 8 \cdot 17 & 8 \cdot 10 & \\ \text { Nitrogen : } & & & 8 \cdot 50\end{array}$

leading to the formula $\mathrm{C}_{20} \mathrm{H}_{13} \mathrm{NO}_{2}$, as may be seen from the following comparison of the theoretical and experimental numbers :-

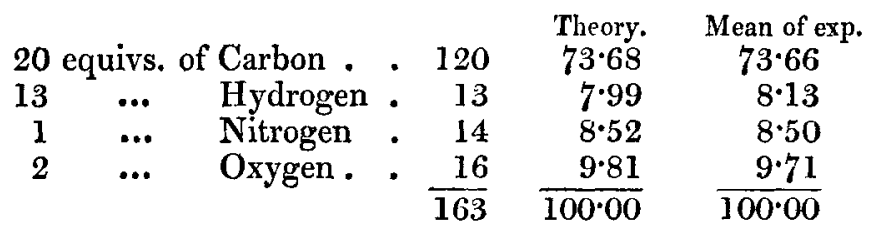

This body therefore is cuminamide, $\mathrm{NH}_{2} \mathrm{C}_{20} \mathrm{H}_{11} \mathrm{O}_{2}$, having:

* In this operation a large quantity of an oily body is produced, which floats on the surface of the hydrochloric acid. It is evidently cumol. 
precisely the same relation to cuminate of ammonia as oxamide to oxalate of ammonia.

In preparing large quantities of this substance the employ-o ment of close tubes would be very inconvenient, and I soon found that it could be obtained in a retort by the continued application of a heat sufficient to keep the salt in a state of semi-fluidity. The analyses II. and III. were made with the product obtained in this manner.

Cuminamide crystallizes like benzamide, in two forms, according to the state of the solution; if crystallized immediately, or from a strong solution, it separates in the form of crystalline tables of great brilliancy, but if the solution be dilute, it crystallizes after the lapse of some hours in long opake needles, both forms having exactly the same composition. It is soluble in hot and cold alcohol in any proportion, as also in ather. This new amide differs from most others that have been described in remaining intact on the addition of strong solution of potash, or mineral acids; from the former it crystallizes in large plates after some days. Long boiling with alkalies or acids is scarcely sufficient to produce the characteristic conversion of amides either into ammoniacal salts or combinations of the base with the acid and evolution of ammonia.

Cumonitrile.-On heating cuminate of ammonia until it is perfectly fused, and keeping the fused mass in a state of brisk ebullition, large globules of a light yellowish oil pass over with water, evidently derived from the decomposition of the salt; when the globules began to diminish the process was stopped, the oil was separated from the water in the receiver by means of a pipette, the remaining distillate added to the mass in the retort, and the process again repeated as before; in this manner, after some half-dozen distillations, nearly an ounce of oil was obtained; it was well-washed with ammonia to remove cuminic acid, which seemed to be soluble in the oil, then treated with hydrochloric acid to remove ammonia, thoroughly washed with water, and digested with chloride of calcium; after standing some days to separate chloride of calcium, it was distilled and carefully rectified, the first portions being rejected, as possibly containing traces of water; the middle portion was rescrved and placed in a retort with a coil of platinum ; the liquid entered into ebuliition at $239^{\circ} \mathrm{C}$., at which point it remained stationary while at least a quarter of an ounce was passing over. This portion was employed in the following analyses:-

I. 0.212 grm. burnt with oxide of copper yielded 0.644 of carbonic acid and $0 \cdot 145$ of water. 
II. 0.225 grm. yielded 0.6835 of carbonic acid and 0.161 of water.

III. $0.244 \mathrm{grm}$. ignited with soda-lime yielded 0.364 of ammonio-chloride of platinum*.

From these analytical results the following per-centages are obtained :-

\begin{tabular}{lrrr} 
& I. & \multicolumn{1}{c}{ II. } & IIl. \\
Carbon . : & $\mathbf{8 2} \cdot 82$ & $82 \cdot 84$ & \\
Hydrogen & $7 \cdot 59$ & $7 \cdot 96$ & \\
Nitrogen : & & & $9 \cdot 34$
\end{tabular}

leading to the formula $\mathrm{C}_{20} \mathrm{H}_{11} \mathrm{~N}$, as may be seen from the following comparison of the theoretical and experimental numbers :-

\begin{tabular}{|c|c|c|c|}
\hline & & Theory. & Mean of exp. \\
\hline 20 Carbon & 120 & $82 \cdot 76$ & $82 \cdot 83$ \\
\hline 11 Hydrogen & 11 & $7 \cdot 58$ & $7 \cdot 77$ \\
\hline 1 Nitrogen & 14 & $9 \cdot 66$ & $9 \cdot 34$ \\
\hline
\end{tabular}

This body is therefore cumonitrile, $\mathrm{C}_{20} \mathrm{H}_{11} \mathrm{~N}$, standing in the same relation to cuminate of ammonia as cyanogen does to oxalate of ammonia.

Cumonitrile is a perfectly clear and colourless liquid, possessing a high refractive power; it has a most powerful and agreeable odour and a burning taste; it is somewhat soluble in water, causing turbidity in that liquid; it is soluble in all proportions of alcohol and æther; it is lighter than water, having a specific gravity 0.765 at $14^{\circ} \mathrm{C}$. (57 Fahr.). The boiling-point, when in contact with metal, is constant at $239^{\circ} \mathrm{C} .\left(462 \cdot 2^{\circ} \mathrm{Fahr}\right.$.), at the barometric pressure $0.7585 \mathrm{~m}$. $=(29.85$ inches $)$. The equivalent of cuminic acid containing $3 \mathrm{C}_{2} \mathrm{H}_{2}$ more than the equivalent of benzoic acid, it was interesting to compare the boiling-points of benzonitrile and cumonitrile. According to Fehling's experiments, the boiling-point of benzonitrile is $191^{\circ} \mathrm{C}$.; on calculating from this observation the boiling-point of cumonitrile according to the rules first pointed out by Kopp, the boiling-point should be $191+3 \cdot 19=248$.

Dr. Fehling does not however mention that he had this substance in contact with metal, and it is not improbable that the true boiling-point of benzonitrile is somewhat lower; the vapour of cumonitrile is very inflammable and burns with a bright flame, which deposits much carbon.

* Professor Fehling found it difficult to estimate the nitrogen in benzonitrile in the form of ammonia, drops of oil passing over into the hydrochloric acid. In the case of cumonitrile, this method gave very accurate results; vil drops also passed over, but they were evidently cumol. 
The strongest nitric acid has but little action upon this substance; after boiling, however, and setting aside for some days, crystals of cuminic acid are formed. On being heated with potassium it darkened, and apparently another oil was produced; the mass on being washed and tested for cyanogen in the usual manner gave a copious precipitate of prussian blue, which seems to be strongly in favour of the view which Kolbe and Frankland have recently promulgated. A strong alcoholic solution of potash has no immediate action on cumonitrile, but after a day or two, on pouring the liquid into a watch-glass, it partially solidified into a yellow crystalline mass, a mixture of the original substance with white crystals. These crystals after purification had all the appearance of cuminamide, and in order to be satisfied of their composition-

I. 0.174 grm. burnt with oxide of copper yielded 0.472 of carbonic acid and $0^{\circ} 124$ of water; the calculated per-centage of carbon and hydrogen from these numbers being-

$$
\begin{array}{lr}
\text { Carbon * } & 73 \cdot 62 \\
\text { Hydrogen } & 7 \cdot 91
\end{array}
$$

These numbers correspond to those of cuminamide, as may be seen by a comparison with the former analyses.

It appears then that cumonitrile, on the addition of potash, is not, as might have been expected, converted into cuminate of ammonia, but into cuminamide, taking 2 instead of 4 atoms of water $-\mathrm{C}_{20} \mathrm{H}_{11} \mathrm{~N}+2 \mathrm{HO}=\mathrm{C}_{20} \mathrm{H}_{13} \mathrm{NO}_{2}$, the latter body being, as before remarked, in such a remarkable degree unaffected by alkalies or acids.

Having obtained one amide with comparative ease, many other ammoniacal salts were heated for the purpose of obtaining analogous amidogen compounds. Benzoate of ammonia was tried unsuccessfully, and it appears from the account published by Fehling of his investigation of benzonitrile, that the residue in the retort consisted entirely of benzoate of ammonia, that salt appearing to have lost directly 4 equivs. of water without undergoing an intermediate conversion into an amide by the loss of 2 equivs. Nitrobenzoic acid was dissolved in ammonia, evaporated, and cautiously fused for a considerable time; when cold it was found to be insoluble in water and ammonia at the ordinary temperature, but dissolved by hot water, from which it crystallized in beautiful yellow needles. On analysis, the following results were obtained :-

I. $0.222 \mathrm{grm}$. of substance burnt with oxide of copper yielded 0.410 of carbonic acid and $0^{\circ} 080$ of water.

II. $0.255 \mathrm{grm}$. yielded 0.472 of carbonic acid and 0.087 of water.

Phil. Mag. S. 3. Vol.31. No. 210. Dec. 184\%. $2 \mathrm{H}$ 
466 On the Decomposition of Cuminate of Ammonia by Heat.

From these results the following per-centages were obtained:-

$$
\begin{array}{rrr}
\text { Carbon . . . } & 50 \cdot 36 & 50 \cdot 43 \\
\text { Hydrogen . . } & 4 \cdot 00 & 3 \cdot 78
\end{array}
$$

corresponding to the formula $\mathrm{C}_{14} \mathrm{H}_{6} \mathrm{~N}_{2} \mathrm{O}_{6}$, as may be seen from the comparison of the theoretical and experimental numbers :-

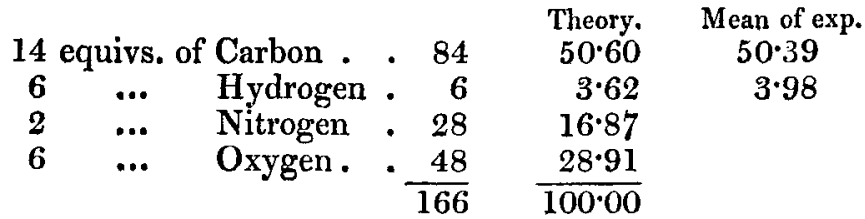

This body is therefore nitrobenzamide, having the same relation to nitrobenzoate of ammonia as cuminamide has to cuminate of ammonia.

This beautiful substance can only be obtained with difficulty, as the nitrobenzoate of ammonia explodes violently unless very great caution is employed.

A specimen of chlorobenzoic acid, made in the laboratory for some other investigation, was dissolved in ammonia and heated; it fused readily, became perfectly insoluble in cold water and ammonia, but soluble in hot water, crystallizing as the solution cooled in long needles of great beauty. The specimen of acid afforded me, being all that could be spared, was insufficient for the manufacture of an amide; I prepared a portion of chlorobenzoic acid by acting upon benzoic acid for some days with hydrochloric acid and chlorate of potash; after purification it was burnt with chromate of lead and gave the following results :-

I. $0.394 \mathrm{grm} .=0 \cdot 769$ of carbonic acid and 0.114 of water.

From this result the following per-centage was obtained :-

$$
\begin{array}{llr} 
& \text { Experiment. } & \text { Theory. } \\
\text { Carbon . . . } 53 \cdot 22 & 53 \cdot 61 \\
\text { Hydrogen . . } 3 \cdot 22 & 3 \cdot 25
\end{array}
$$

leading to the formula $\mathrm{HO}, \mathrm{C}_{14}\left\{\begin{array}{l}\mathrm{H}_{4} \\ \mathrm{Cl}\end{array}\right\} \mathrm{O}_{3}, 1$ equiv. of the hydrogen of benzoic acid replaced by an equivalent of chlorine.

This acid, however, on being subjected to the usual treatment by solution in ammonia and subsequent heat, did not fuse but blackened, charcoal being separated. Unfortunately the specimen of ammoniacal salt from which $I$ had made the former compound was not analysed, probably it would have 
proved to be $\mathrm{C}_{14}\left\{\begin{array}{l}\mathrm{H}_{3} \\ \mathrm{Cl}_{2}\end{array}\right\} \mathrm{O}_{3}, \mathrm{HO}$, or $\mathrm{C}_{14}\left\{\begin{array}{l}\mathrm{H}_{2} \\ \mathrm{Cl}_{3}\end{array}\right\} \mathrm{O}_{3}, \mathrm{HO}, \mathrm{a}$ dichlorobenzoic or a trichlorobenzoic acid, such existing.

These experiments were conducted in the laboratories of the Royal College of Chemistry under the direction of Dr. Hofmann, to whom I beg to offer my best thanks for his advice and assistance during their progress.

LXXI. On the General Solution (in certain cases) of the equation $x^{3}+y^{3}+\mathbf{A} z^{3}=\mathbf{M} x y z, \& c . \quad B y$ J. J. Sycvester, A.M., F.R.S., late Professor of Natural Philosophy in University College, London*.

I SHALL restrict the enunciation of the proposition I am about to advance to much narrower limits than I believe are necessary to the truth, with a view to avoid making any statement which I may hereafter have occasion to modify. Let us then suppose in the equation

$$
x^{3}+y^{3}+\mathrm{A} z^{3}=\mathrm{M} x y z
$$

that $\mathrm{A}$ is a prime number, and that $27 \mathrm{~A}-\mathrm{M}^{3}$ is positive, but exempt from positive prime factors of the form $6 i+1$. Then I say, and have succeeded in demonstrating, that all the possible solutions in integer numbers of the given equation may be obtained by explicit processes from one particular solution or system of values of $x, y, z$, which may be called the Primitive system.

This system of roots or of values of $x, y, z$ is that system in which the value of the greatest of the three terms $x, y, \mathrm{~A}^{\frac{1}{s}} . z$ (which may be called the Dominant) is the least possible of all such dominants. I believe that in general the system of the least Dominant is identical with the system of the least Content, meaning by the latter term the product of the three terms out of which the Dominant is elected. I proceed to show the law of derivation.

To express this simply, I must premise that I shall have to employ such an expression as $S^{\prime}=\phi(S)$ to indicate, not that a certain quantity, $S^{\prime}$, is a function of $S$, but that a certain system of quantities disconnected from one another, denoted by $S^{\prime}$, are severally functions of a certain other system of quantities denoted by $S$; and, as usual, I shall denote $\phi \phi S$ by $\phi^{2} S, \phi \phi^{2} \mathrm{~S}$ by $\phi^{3} \mathrm{~S}$, and so forth.

Let now $\mathrm{P}$ be the Primitive system of solution of the equation

$$
x^{3}+y^{3}+\mathrm{A} z^{3}=\mathrm{M} x y z
$$

* Communicated by the Author.

$2 \mathrm{H} 2$ 